\title{
Transformed Contribution Ratio Test for the Number of Factors in Static Approximate Factor Models
}

\author{
Qiang Xia ${ }^{\mathrm{a}}$, Rubing Liang ${ }^{\mathrm{a}, *}$, Jianhong $\mathrm{Wu}^{\mathrm{b}}$ \\ ${ }^{a}$ College of Mathematics and Informatics, South China Agricultural University, Guangzhou \\ 510642, China \\ ${ }^{b}$ School of Mathematics and Science, Shanghai Normal University, Shanghai 200234, China
}

\begin{abstract}
Determining the number of factors $(r)$ is of importance in static approximate factor models. Under some mild conditions, the $r$ largest eigenvalues of the variance matrix of $N$ response variables go to infinity as $N$ increases, while the rest are bounded. Then, "Eigenvalue Ratio" (ER) and "Growth Ratio" (GR) estimators have been well exploited by maximizing the ratio of two adjacen$\mathrm{t}$ eigenvalues in the literature. As a modification of ER and GR estimators, the new estimator named as "Transformed Contribution Ratio" (TCR) is obtained by maximizing the ratio of two adjacent transformed contribution of the eigenvalues. Under the same conditions of ER and GR estimators, the resulted estimator can be proved to be consistent. It can be further shown that, comparing with the competitors in the existing literature, the new method has desired performance on truly selecting the value of the number of latent common factors, especially when both strong and weak factors or some dominant factors are in static approximate factor models. Monte Carlo simulation experiments and one real data application are carried out for illustration.
\end{abstract}

Keywords: Approximate factor model, Eigenvalues, Number of factors, Transformed contribution ratio

\footnotetext{
${ }^{*}$ Corresponding author: Rubing Liang, College of Mathematics and Informatics, South China Agricultural University, Guangzhou 510642. Email: stat2003@163.com. Tel. and fax: +8685285393 .
}

Preprint submitted to Computational Statistics and Data Analysis

January 7, 2017

(C) 2017. This manuscript version is made available under the Elsevier user license http://www.elsevier.com/open-access/userlicense/1.0/ 


\section{Introduction}

In the analysis of large dimensional factor models, one of fundamental issues is the consistent determination of the number of common factors. Recently, attempts in this direction have been focused on this issue in economic and financial data with both large cross-section dimension $(\mathrm{N})$ and time series observations (T). Many examples include but not limited to Bai and Ng (2002), Stock and Watson (2002), Onatski (2006, 2010), Alessi et al. (2010), Fan et al. (2013) and Ahn and Horenstein (2013) for static approximate factor models of Chamberlain and Rothschild (1983). Pan and Yao (2008), Lam and Yao (2012), Xia et al. (2015) focused on the determination of the number of factors for highdimensional time series. Much of the other related econometric research, such as Forni et al. (2000), Hallin and Liska (2007), Amengual and Watson (2007), Bai and Ng (2007), and Onatski (2009), among others, were papers on dynamic factor models.

These estimators, however, are not directly comparable as they are based on their different sets of hypothesis. Meanwhile, under the same predetermined conditions, various methods can lead to different estimation results for the number of factors. In this paper, we will improve eigenvalue-based ratio-type method for the number of common factors suggested by Ahn and Horenstein (2013) in static approximate factor models, the improved method can be expected to have desired performance comparing with the existing methods in the literature. Up to our knowledge, Bai and Ng (2002) proposed to estimate the number of factors in static approximate factor models by minimizing the information criteria of model selection, named PC and IC. But the criteria of Bai and Ng (2002) are known to overestimate in practice. Onatski (2006) obtained a consistent estimator of the $(r+1)$ th largest eigenvalue and then the corresponding threshold value can be found easily only if it is slightly larger than the $(r+1)$ th largest eigenvalue, where $r$ is the true number of common factors. However, the method is available only under the assumption that the idiosyncratic errors are either autocorrelated or cross-sectionally correlated, but not both (Ahn and 
Horenstein, 2013). Onatski (2010) considered the so-called "Edge Distribution" estimator by using the differenced eigenvalues. Alessi et al. (2010) introduced a tuning multiplicative constant in the penalty to improve the method of Bai and $\mathrm{Ng}$ (2002), an idea that was proposed by Hallin and Liska (2007) in the context of dynamic factor models. As reported by Ahn and Horenstein (2013), the methods proposed by Bai and Ng (2002) and Onatski (2010) have worse finite sample properties in the case with cross-sectional dependency although they do perform well in the case with independent idiosyncratic errors. Two eigenvaluesbased ratio-type estimators of Ahn and Horenstein (2013) were proposed and shown to perform well even when the idiosyncratic errors are cross-sectionally dependent or serially correlated. Lam and Yao (2012) used the similar idea to deal with the factor modeling for high-dimensional time series based on the dimension-reduction viewpoint. As argued by Ahn and Horenstein (2013), almost all the above estimators need to predetermine the possible maximum of the number of factors, which may lead to overestimation or underestimation of the number when the maximum (hereafter, denoted by $k$ max) is too large or too small. But, the ER and GR estimators are not sensitive to kmax by the simulation experiments of Ahn and Horenstein (2013).

This paper proposes a determination approach by improving eigenvaluebased ratio-type method for the number of common factors in static approximate factor models. According to Ahn and Horenstein (2013), the ER estimator performs well under the cases of strong factors, while the GR estimator outperforms the ER estimator in the case when both strong and weak factors or some dominant factors are in static approximate factor models. The new method named as "Transformed Contribution Ratio" (hereafter TCR) is based on the ratio values of adjacent transformed contribution of eigenvalues arranged in descending order, and expected to have both advantages of the ER and GR estimators. Under some mild conditions, the resulted estimator can be proved to be consistent. It can be further shown that, comparing with the competitors ${ }_{60}$ in the existing literature, the new estimator has desired performance on truly selecting the value of the number of latent common factors, especially when 
both strong and weak factors or some dominant factors are in static approximate factor models. Monte Carlo simulation experiments and one real data application are carried out for illustration.

The rest of this paper is organized as follows. In Section 2, we introduce the new method of determining the number of common factors in the approximate factor models and state its asymptotic consistency. Section 3 carries out some simulation experiments to examine the finite sample performance of the new estimation. One real data analysis for illustration are given in Section 4. Section 5 provides some concluding comments. Technical details on the proofs of theorems are described in the Appendix.

Throughout this paper, $A^{\prime}$ means the transpose of the matrix $A,\|A\|=$ $\left[\operatorname{trace}\left(A^{\prime} A\right)\right]^{1 / 2}$ denotes the norm of the matrix $A$, and $\psi_{i}(\Omega)$ denotes the $i$ th largest eigenvalue of a positive semi-definite matrix $\Omega$.

\section{Estimation of the Number of Factors}

Consider the following static approximate factor model of Chamberlain and Rothschild (1983),

$$
y_{\cdot t}=\Lambda F_{t}+u_{\cdot t}, \quad t=1,2, \ldots, T,
$$

where $y_{\cdot t}=\left(y_{1 t}, \ldots, y_{N t}\right)^{\prime}, F_{t}$ is an $r$-dimensional vector of common factors, $\Lambda=\left(\lambda_{1}, \lambda_{2}, \ldots, \lambda_{N}\right)^{\prime}, \lambda_{i}$ is an $r$-dimensional vector of factor loadings, and $u_{\cdot t}=$

so $\left(u_{1 t}, \ldots, u_{N t}\right)^{\prime}$ is the vector of the idiosyncratic error. The number of factors $(r)$ is unknown and needs to be estimated. For the sake of statement, we denote that $Y=\left(y \cdot 1, \ldots, y_{T}\right)^{\prime}$ is the $T \times N$ observation matrix with the element $y_{i t}$, $F=\left(F_{1}, F_{2}, \ldots, F_{T}\right)^{\prime}$, and $U=\left(u_{\cdot 1}, \ldots, u \cdot T\right)^{\prime}$ is the $T \times N$ idiosyncratic error matrix with the element $u_{i t}, i=1, \ldots, N, t=1, \ldots, T$. And then, model (1) 85 can be rewritten as the following matrix form

$$
Y=F \Lambda^{\prime}+U
$$

Let

$$
\tilde{\mu}_{N T, i}=: \psi_{i}\left(Y^{\prime} Y /(N T)\right)=\psi_{i}\left(Y Y^{\prime} /(N T)\right)
$$


denote the $i$-th largest eigenvalue of $Y^{\prime} Y /(N T)$. Ahn and Horenstein (2013) found that the first $r$ eigenvalues are $O_{p}(1)$ and the rest are at most $O_{p}\left(\frac{1}{m}\right)$, and then proposed the eigenvalue-based ratio-type estimators, i.e., ER and GR, to determine the number of common factors as follows

$$
\hat{r}_{E R}=\arg \max _{1 \leq i \leq k \max } \frac{\tilde{\mu}_{N T, i}}{\tilde{\mu}_{N T, i+1}}, \quad \hat{r}_{G R}=\arg \max _{1 \leq i \leq k \max } \frac{\ln \left(V_{i-1}\right)-\ln \left(V_{i}\right)}{\ln \left(V_{i}\right)-\ln \left(V_{i+1}\right)},(4)
$$

where $m=\min \{N, T\}$ and $M=\max \{N, T\}, V_{i}=\sum_{j=i+1}^{m} \tilde{\mu}_{N T, j}$, and kmax is the predetermined possible maximum value of the number of factors.

Ahn and Horenstein (2013) argued that the two objective functions in (4) at the true value point of the number of factors are $O_{p}(m)$ and the others $O_{p}(1)$, and then the eigenvalue ratio-based estimators can be proven to be consisten$\mathrm{t}$ when $m$ is sufficiently large. In practice, however, when not all factors are strong, the number of common factors is not detected accurately and can be underestimated. In order to avoid this problem, we can use the modified objective function to replace those of (4) suggested by Ahn and Horenstein (2013), and then obtain the estimator $\hat{r}_{T C R}$ as follows.

$$
\begin{aligned}
\hat{r}_{T C R} & =\arg \max _{1 \leq i \leq k \max } \frac{\ln \left(V_{i-1}+\tilde{\mu}_{N T, i}\right)-\ln \left(V_{i-1}\right)}{\ln \left(V_{i}+\tilde{\mu}_{N T, i+1}\right)-\ln \left(V_{i}\right)} \\
& =\arg \max _{1 \leq i \leq k \max } \frac{\ln \left(1+\tilde{\mu}_{N T, i} / V_{i-1}\right)}{\ln \left(1+\tilde{\mu}_{N T, i+1} / V_{i}\right)},
\end{aligned}
$$

Clearly, this transformation in the numerator of the objective function in (6) includes the main part $\tilde{\mu}_{N T, i} / V_{i-1}$, which measures $\tilde{\mu}_{N T, i}$ 's influence on $V_{i-1}$, and can be called the contribution of $\tilde{\mu}_{N T, i}$ for $V_{i-1}$. Then, $\ln \left(1+\tilde{\mu}_{N T, i} / V_{i-1}\right)$ is the transformed contribution. Meanwhile, as a modification of ER and GR estimators in (5) or (6), we name it as "Transformed Contribution Ratio" (hereafter TCR) estimator.

Remark 1. Ahn and Horenstein (2013) argued that the ER and GR estimators are not sensitive to the choice of kmax unless it is too large or too small. However, when both strong and weak factors or some dominant factors are existent, the ratio value of two adjacent eigenvalues may be not stable because the positive eigenvalue $\tilde{\mu}_{N T, i}$ is very small or very large. (5) is similar to the 
ER estimator in fact, only with the ln-difference versus the difference between $V_{i-1}$ and $V_{i}$ in the numerator of (5). From (6), due to $\ln \left(1+\tilde{\mu}_{N T, i} / V_{i-1}\right)<$ $\tilde{\mu}_{N T, i} / V_{i-1}$ for $\tilde{\mu}_{N T, i} / V_{i-1}>0$, when $\tilde{\mu}_{N T, i}$ is large, the use of the transformation $\ln \left(1+\tilde{\mu}_{N T, i} / V_{i-1}\right)$ is a shrinkage to the large values $\tilde{\mu}_{N T, i} / V_{i-1}$. Moreover, the main role of $\tilde{\mu}_{N T, i} / V_{i-1}$ is measuring $\tilde{\mu}_{N T, i}$ 's effect, which is not only meaningful but also useful for TCR to avoid the underestimation of the number of common factors. Especially when the dominant factors or weak factors exist, TCR will perform well, see the simulation study in Section 3 for more details.

By Ahn and Horenstein (2013), the above objective function in (5) or (6) can be expected to guarantee the consistency of the estimation under the same conditions of Ahn and Horenstein (2013), which are as follows.

Assumption A: Let $\mu_{N T, i}=\psi_{i}\left(\left(\Lambda^{\prime} \Lambda / N\right)\left(F^{\prime} F / T\right)\right)$ for $i=1, \ldots, r$. Then, for each $i=1, \ldots, r, \operatorname{plim}_{m \rightarrow \infty} \mu_{N T, i}=\mu_{i} \in(0,+\infty)$. Moreover, the number $r$ of common factors is assumed to finite.

\section{Assumption B:}

(i) $E\left\|F_{t}\right\|^{4} \leq c_{1}, E\left\|\lambda_{i}\right\|^{4} \leq c_{1}$, for all $N$ and $T$.

(ii) $E\left(\left\|N^{-\frac{1}{2}} \sum_{i=1}^{T} u_{i t} \lambda_{i}\right\|^{2}\right) \leq c_{1}$ for all $t$.

(iii) $E\left(N^{-1} \sum_{i=1}^{N}\left\|T^{-\frac{1}{2}} \sum_{t=1}^{T} u_{i t} F_{t}\right\|^{2}\right)=E\left[(N T)^{-1}\left\|U^{\prime} F\right\|\right] \leq c_{1}$.

Assumption C: $\quad \psi_{1}\left(U^{\prime} U / M\right)=O_{p}(1)$, and $\psi_{\left[d^{c} m\right]}\left(U^{\prime} U / M\right) \geq c+o_{p}(1)$ for some positive and finite real number $c$ and some $d^{c} \in(0,1]$.

Remark 2. The above three conditions are the same as weak conditions of Ahn and Horenstein (2013). $\psi_{1}\left(U^{\prime} U / M\right)=O_{p}(1)$ in Assumption $C$ makes clear that the error matrix $U$ does not contain common factors. $\psi_{\left[d^{c} m\right]}\left(U^{\prime} U / M\right) \geq c+o_{p}(1)$ indicates that the first largest $\left[d^{c} M\right]$ eigenvalues of $U^{\prime} U / M$ are bounded away from zero, which can guarantee the consistency of the TCR estimator.

Theorem 1. Suppose that Assumptions A-C hold with $r>1$, we then have

$$
\lim _{m \rightarrow \infty} \operatorname{Pr}\left(\hat{r}_{T C R}=r\right)=1, \quad \text { for any } k \max \in\left(r,\left[d^{c} m-r-1\right]\right) .
$$


In the case with no factors, i.e., $r=0$, we can slightly modify the above estimator by the same method as that of Ahn and Horenstein (2013). Specifically, we can define a mock eigenvalue, e.g., $\tilde{\mu}_{N T, 0}=V_{0} / \ln (m)$ such that $\tilde{\mu}_{N T, 0} \rightarrow 0$ and $m \tilde{\mu}_{N T, 0} \rightarrow \infty$ as $m \rightarrow \infty$, although a finite multiple of the choice is also available.

Then, we have Corollary 1 as follows.

Corollary 1. Suppose that Assumptions $A$-C hold, redefine $\hat{r}_{T C R}$ using $\tilde{\mu}_{N T, 0}$ for $i=0$, then it follows that

$$
\lim _{m \rightarrow \infty} \operatorname{Pr}\left(\hat{r}_{T C R}=r\right)=1
$$

where $\hat{r}_{T C R}=\arg \max _{0 \leq i \leq k \max } \frac{\ln \left(1+\tilde{\mu}_{N T, i} / V_{i-1}\right)}{\ln \left(1+\tilde{\mu}_{N T, i+1} / V_{i}\right)}$.

\section{Simulation Study}

In this section, some simulation experiments are carried out to investigate the performance of the suggested estimator $\hat{r}_{T C R}$. To compare with the competitors, the foundation of our simulation exercises is the model of Ahn and Horenstein (2013):

$$
y_{i t}=\sum_{j=1}^{r} \lambda_{i j} F_{j t}+\sqrt{\theta} u_{i t}, \quad u_{i t}=\sqrt{\frac{1-\rho^{2}}{1+2 J \beta^{2}}} e_{i t},
$$

where $e_{i t}=\rho e_{i, t-1}+(1-\beta) v_{i t}+\sum_{l=\max \{i-J, 1\}}^{\min \{i+J, n\}} \beta v_{l t}$, and $\lambda_{i j}$ and $v_{l t}$ are both drawn from the standard normal distribution $N(0,1)$. The factors $F_{j t}$ are drawn from normal distribution with zero means.

In the simulation experiments, the settings of some parameters are important to show the various structure of data. The control parameter $\theta$ is the inverse of the signal to noise ratio $(S N R)$ of each factor, i.e., $S N R=\frac{1}{\theta}=\frac{\operatorname{var}\left(F_{j t}\right)}{\operatorname{var}\left(\sqrt{\theta} u_{i t}\right)}$ with $\operatorname{var}\left(F_{j t}\right)=1$. Then, we can adjust the value of $\theta$ while fixing variances of factors at 1 . Moreover, $\rho$ can show the serial correlation of the idiosyncratic errors over the time index, and $\beta$ and $J$ can control the cross-sectional dependency of the idiosyncratic errors over the individual index. 
Our simulations are categorized into four parts with the same settings as Ahn and Horenstein (2013). The performances of TCR estimator $\left(\hat{r}_{T C R}\right)$ are compared with those of the ER and GR estimators $\left(\hat{r}_{E R}, \hat{r}_{G R}\right)$ of Ahn and Horenstein (2013), the BIC3 estimator $\left(\hat{r}_{B N}\right)$ of Bai and $\mathrm{Ng}(2002)$, the ED estimator $\left(\hat{r}_{E D}\right)$ of Onatski (2010), and the IC1 estimator $\left(\hat{r}_{A B C}\right)$ of Alessi et al. (2010). The estimating procedure for the number $r$ is replicated 1000 times, and the corresponding results are denoted by $x(y \mid z)$, where $x$ is the mean of different estimators in the 1000 replications, the numbers $y$ and $z$ in parentheses $(y \mid z)$ are the number of underestimation and overestimation in 1000 the replications, respectively, and then $1000-y-z$ is the correct number of estimation.

The first part is designed to investigate four cases with different error structures in the following, which will influence the finite-sample performances of TCR estimator as well as the other estimators:

$\left(C_{1}\right)$ independent and identically distributed errors $(\rho=\beta=J=0)$;

$\left(C_{2}\right)$ serially correlated errors $(\rho=0.7, \beta=J=0)$;

$\left(C_{3}\right)$ cross-sectionally correlated errors $(\rho=0, \beta=0.5, J=\max \{10, N / 20\})$;

$\left(C_{4}\right)$ both serially and cross-sectionally correlated errors $(\rho=0.5, \beta=$ $0.2, J=\max \{10, N / 20\})$.

Table 1 reports the results of the six estimators when three factors $(r=3)$ are drawn from $N(0,1)$ and $\theta$ is fixed at 1 . In the cases with i.i.d. errors $\left(C_{1}\right)$, the results show that $\hat{r}_{T C R}$ and $\hat{r}_{A B C}$ are slightly better than the other estimators with $N=T=25$; when $N=T \geq 50$, all the six estimators $\hat{r}_{E R}, \hat{r}_{G R}$, $\hat{r}_{B N}, \hat{r}_{A B C}, \hat{r}_{E D}$ and $\hat{r}_{T C R}$ have the desired performance. For the data with serially correlated errors $\left(C_{2}\right), \hat{r}_{E D}$ is slightly better than the other estimators with $N=T=25$; when $N=T \geq 50, \hat{r}_{T C R}$ performs equally to or better than the other estimators. For the data with cross-sectionally correlated errors $\left(C_{3}\right)$, all estimators have the bad performance in the cases with $N=T=25,50$; when $N=T=75, \hat{r}_{E R}$ outperforms the other estimators; when $N=T \geq 100$, all estimators excluding $\hat{r}_{B N}$ have the good performance. In the case with serially/ cross-sectionally correlated errors $\left(C_{4}\right), \hat{r}_{E R}$ is still better than the 
other estimators with $N=T=25,50$; when $N=T \geq 75, \hat{r}_{T C R}$ performs as well as the $\hat{r}_{E R}, \hat{r}_{G R}$ estimators, which all outperform the other estimators. It appears that the performance of the $\hat{r}_{B N}$ estimator is much more sensitive to cross-sectional correlation than autocorrelation in the errors from the cases $C_{3}$ $C_{4}$, which are the same as the results reported by Ahn and Horenstein (2013).

Table 1: Effects of error covariance structure with three factors

\begin{tabular}{|c|c|c|c|c|c|c|c|c|c|}
\hline Case & $N$ & $T$ & $r$ & $\hat{r}_{E R}$ & $\hat{r}_{G R}$ & $\hat{r}_{B N}$ & $\hat{r}_{A B C}$ & $\hat{r}_{E D}$ & $\hat{r}_{T C R}$ \\
\hline \multirow{8}{*}{$C_{1}$} & 25 & 25 & 3 & $2.895(75 \mid 0)$ & $2.974(21 \mid 0)$ & $3.163(0 \mid 158)$ & $2.994(7 \mid 6)$ & $3.029(0 \mid 23)$ & $2.991(9 \mid 0)$ \\
\hline & 50 & 50 & 3 & $3.000(0 \mid 0)$ & $3.000(0 \mid 0)$ & $3.000(0 \mid 0)$ & $3.001(0 \mid 1)$ & $3.011(0 \mid 9)$ & $3.000(0 \mid 0)$ \\
\hline & 75 & 75 & 3 & $3.000(0 \mid 0)$ & $3.000(0 \mid 0)$ & $3.000(0 \mid 0)$ & $3.000(0 \mid 0)$ & $3.002(0 \mid 2)$ & $3.000(0 \mid 0)$ \\
\hline & 100 & 100 & 3 & $3.000(0 \mid 0)$ & $3.000(0 \mid 0)$ & $3.000(0 \mid 0)$ & $3.000(0 \mid 0)$ & $3.009(0 \mid 5)$ & $3.000(0 \mid 0)$ \\
\hline & 125 & 125 & 3 & $3.000(0 \mid 0)$ & $3.000(0 \mid 0)$ & $3.000(0 \mid 0)$ & $3.000(0 \mid 0)$ & $3.003(0 \mid 2)$ & $3.000(0 \mid 0)$ \\
\hline & 150 & 150 & 3 & $3.000(0 \mid 0)$ & $3.000(0 \mid 0)$ & $3.000(0 \mid 0)$ & $3.000(0 \mid 0)$ & $3.005(0 \mid 5)$ & $3.000(0 \mid 0)$ \\
\hline & 175 & 175 & 3 & $3.000(0 \mid 0)$ & $3.000(0 \mid 0)$ & $3.000(0 \mid 0)$ & $3.000(0 \mid 0)$ & $3.013(0 \mid 8)$ & $3.000(0 \mid 0)$ \\
\hline & 200 & 200 & 3 & $3.000(0 \mid 0)$ & $3.000(0 \mid 0)$ & $3.000(0 \mid 0)$ & $3.000(0 \mid 0)$ & $3.002(0 \mid 2)$ & $3.000(0 \mid 0)$ \\
\hline \multirow{8}{*}{$C_{2}$} & 25 & 25 & 3 & $2.163(765 \mid 40)$ & $2.607(598 \mid 112)$ & $6.800(0 \mid 1000)$ & $2.833(456 \mid 157)$ & $3.294(357 \mid 300)$ & $2.826(517 \mid 183)$ \\
\hline & 50 & 50 & 3 & $2.997(3 \mid 0)$ & $3.000(0 \mid 0)$ & $4.494(0 \mid 955)$ & $3.142(0 \mid 89)$ & $3.072(0 \mid 46)$ & $3.000(0 \mid 0)$ \\
\hline & 75 & 75 & 3 & $3.000(0 \mid 0)$ & $3.000(0 \mid 0)$ & $3.073(0 \mid 72)$ & $3.048(0 \mid 36)$ & $3.033(0 \mid 19)$ & $3.000(0 \mid 0)$ \\
\hline & 100 & 100 & 3 & $3.000(0 \mid 0)$ & $3.000(0 \mid 0)$ & $3.000(0 \mid 0)$ & $3.030(0 \mid 17)$ & $3.008(0 \mid 8)$ & $3.000(0 \mid 0)$ \\
\hline & 125 & 125 & 3 & $3.000(0 \mid 0)$ & $3.000(0 \mid 0)$ & $3.000(0 \mid 0)$ & $3.009(0 \mid 9)$ & $3.007(0 \mid 7)$ & $3.000(0 \mid 0)$ \\
\hline & 150 & 150 & 3 & $3.000(0 \mid 0)$ & $3.000(0 \mid 0)$ & $3.000(0 \mid 0)$ & $3.003(0 \mid 3)$ & $3.009(0 \mid 5)$ & $3.000(0 \mid 0)$ \\
\hline & 175 & 175 & 3 & $3.000(0 \mid 0)$ & $3.000(0 \mid 0)$ & $3.000(0 \mid 0)$ & $3.000(0 \mid 0)$ & $3.005(0 \mid 5)$ & $3.000(0 \mid 0)$ \\
\hline & 200 & 200 & 3 & $3.000(0 \mid 0)$ & $3.000(0 \mid 0)$ & $3.000(0 \mid 0)$ & $3.000(0 \mid 0)$ & $3.005(0 \mid 3)$ & $3.000(0 \mid 0)$ \\
\hline \multirow{8}{*}{$C_{3}$} & 25 & 25 & 3 & $4.942(1 \mid 998)$ & $4.986(0 \mid 1000)$ & $6.997(0 \mid 1000)$ & $5.123(0 \mid 1000)$ & $5.169(0 \mid 1000)$ & $5.002(0 \mid 1000)$ \\
\hline & 50 & 50 & 3 & $6.615(1 \mid 976)$ & $6.900(0 \mid 1000)$ & $7.004(0 \mid 1000)$ & $7.00(0 \mid 1000)$ & $7.017(0 \mid 1000)$ & $6.940(0 \mid 1000)$ \\
\hline & 75 & 75 & 3 & $3.113(303 \mid 157)$ & $6.132(41 \mid 672)$ & $8.000(0 \mid 1000)$ & $3.963(213 \mid 330)$ & $5.921(120 \mid 693)$ & $6.338(31 \mid 711)$ \\
\hline & 100 & 100 & 3 & $2.999(1 \mid 0)$ & $2.999(1 \mid 0)$ & $8.000(0 \mid 1000)$ & $3.046(0 \mid 15)$ & $3.040(0 \mid 11)$ & $3.000(0 \mid 0)$ \\
\hline & 125 & 125 & 3 & $3.000(0 \mid 0)$ & $3.000(0 \mid 0)$ & $8.000(0 \mid 1000)$ & $3.015(0 \mid 6)$ & $3.002(0 \mid 0)$ & $3.000(0 \mid 0)$ \\
\hline & 150 & 150 & 3 & $3.000(0 \mid 0)$ & $3.000(0 \mid 0)$ & $8.000(0 \mid 1000)$ & $3.004(0 \mid 3)$ & $3.001(0 \mid 1)$ & $3.000(0 \mid 0)$ \\
\hline & 175 & 175 & 3 & $3.000(0 \mid 0)$ & $3.000(0 \mid 0)$ & $8.000(0 \mid 1000)$ & $3.001(0 \mid 1)$ & $3.001(0 \mid 1)$ & $3.000(0 \mid 0)$ \\
\hline & 200 & 200 & 3 & $3.000(0 \mid 0)$ & $3.000(0 \mid 0)$ & $8.000(0 \mid 1000)$ & $3.001(0 \mid 1)$ & $3.001(0 \mid 1)$ & $3.000(0 \mid 0)$ \\
\hline \multirow{8}{*}{$C_{4}$} & 25 & 25 & 3 & $3.466(253 \mid 532)$ & $4.177(103 \mid 747)$ & $6.522(0 \mid 1000)$ & $4.703(44 \mid 889)$ & $4.749(11 \mid 934)$ & $4.386(63 \mid 826)$ \\
\hline & 50 & 50 & 3 & $3.556(39 \mid 261)$ & $4.571(2 \mid 555)$ & $6.203(0 \mid 1000)$ & $6.301(2 \mid 986)$ & $5.787(7 \mid 873)$ & $5.036(1 \mid 689)$ \\
\hline & 75 & 75 & 3 & $3.015(1 \mid 10)$ & $3.142(0 \mid 39)$ & $6.960(0 \mid 1000)$ & $5.510(0 \mid 774)$ & $5.120(0 \mid 464)$ & $3.420(0 \mid 69)$ \\
\hline & 100 & 100 & 3 & $3.000(0 \mid 0)$ & $3.009(0 \mid 2)$ & $7.361(0 \mid 1000)$ & $4.100(0 \mid 384)$ & $3.162(0 \mid 46)$ & $3.009(0 \mid 2)$ \\
\hline & 125 & 125 & 3 & $3.000(0 \mid 0)$ & $3.000(0 \mid 0)$ & $7.442(0 \mid 1000)$ & $3.380(0 \mid 177)$ & $3.019(0 \mid 12)$ & $3.000(0 \mid 0)$ \\
\hline & 150 & 150 & 3 & $3.000(0 \mid 0)$ & $3.000(0 \mid 0)$ & $7.287(0 \mid 1000)$ & $4.106(0 \mid 111)$ & $3.012(0 \mid 7)$ & $3.000(0 \mid 0)$ \\
\hline & 175 & 175 & 3 & $3.000(0 \mid 0)$ & $3.000(0 \mid 0)$ & $7.133(0 \mid 1000)$ & $3.155(0 \mid 71)$ & $3.007(0 \mid 7)$ & $3.000(0 \mid 0)$ \\
\hline & 200 & 200 & 3 & $3.000(0 \mid 0)$ & $3.000(0 \mid 0)$ & $6.984(0 \mid 1000)$ & $3.017(0 \mid 28)$ & $3.001(0 \mid 1)$ & $3.000(0 \mid 0)$ \\
\hline
\end{tabular}


$0.2, J=\max \{10, N / 20\}$

In the second part, the effects of weak factors on the estimators are examined by considering two cases. The first is the case in which all three factors have weak explanatory power $(S N R=0.17)$. The second is the case in which two factors are strong $(S N R=1)$ and one factor is weak $(S N R<1)$.

Table 2: Effects of all weak factors with serially/cross-sectionally correlated

errors

\begin{tabular}{|c|c|c|c|c|c|c|c|c|}
\hline$N$ & $T$ & $r$ & $\hat{r}_{E R}$ & $\hat{r}_{G R}$ & $\hat{r}_{B N}$ & $\hat{r}_{A B C}$ & $\hat{r}_{E D}$ & $\hat{r}_{T C R}$ \\
\hline 25 & 25 & 3 & $2.902(439 \mid 384)$ & $3.537(310 \mid 535)$ & $6.413(0 \mid 1000)$ & $2.528(570 \mid 327)$ & $2.868(425 \mid 437)$ & $3.750(256 \mid 582)$ \\
\hline 50 & 50 & 3 & $5.412(94 \mid 868)$ & $5.912(44 \mid 943)$ & $6.081(0 \mid 1000)$ & $5.365(165 \mid 823)$ & $4.168(404 \mid 591)$ & $6.036(31 \mid 958)$ \\
\hline 75 & 75 & 3 & $5.755(67 \mid 893)$ & $7.124(13 \mid 982)$ & $6.797(0 \mid 1000)$ & $4.195(424 \mid 572)$ & $3.700(594 \mid 406)$ & $7.185(10 \mid 988)$ \\
\hline 100 & 100 & 3 & $4.065(326 \mid 573)$ & $5.888(152 \mid 798)$ & $7.144(0 \mid 1000)$ & $1.491(906 \mid 84)$ & $1.084(984 \mid 6)$ & $5.922(147 \mid 804)$ \\
\hline 125 & 125 & 3 & $2.910(469 \mid 275)$ & $4.137(317 \mid 479)$ & $7.323(0 \mid 1000)$ & $1.452(888 \mid 63)$ & $1.126(955 \mid 4)$ & $4.253(295 \mid 498)$ \\
\hline 150 & 150 & 3 & $2.963(331 \mid 219)$ & $3.563(244 \mid 336)$ & $7.231(0 \mid 1000)$ & $1.524(800 \mid 38)$ & $1.221(914 \mid 3)$ & $3.609(238 \mid 347)$ \\
\hline 175 & 175 & 3 & $2.692(338 \mid 62)$ & $2.919(270 \mid 102)$ & $7.030(0 \mid 1000)$ & $2.284(457 \mid 38)$ & $1.772(667 \mid 8)$ & $2.965(253 \mid 111)$ \\
\hline 200 & 200 & 3 & $2.820(138 \mid 26)$ & $2.897(109 \mid 39)$ & $6.860(0 \mid 1000)$ & $2.700(184 \mid 30)$ & $2.334(342 \mid 6)$ & $2.917(99 \mid 15)$ \\
\hline
\end{tabular}

Table 3: Effects of strong and weak factors with serially/cross-sectionally

correlated errors

\begin{tabular}{cccccccc}
\hline$S N R_{3}$ & $r$ & $\hat{r}_{E R}$ & $\hat{r}_{G R}$ & $\hat{r}_{B N}$ & $\hat{r}_{A B C}$ & $\hat{r}_{E D}$ & $\hat{r}_{T C R}$ \\
\hline 0.45 & 3 & $2.992(4 \mid 0)$ & $3.000(0 \mid 0)$ & $7.329(0 \mid 1000)$ & $4.064(0 \mid 394)$ & $3.210(0 \mid 63)$ & $3.000(0 \mid 0)$ \\
0.40 & 3 & $3.000(0 \mid 0)$ & $3.000(0 \mid 0)$ & $7.300(0 \mid 1000)$ & $4.055(0 \mid 398)$ & $3.126(0 \mid 49)$ & $3.000(0 \mid 0)$ \\
0.35 & 3 & $2.998(1 \mid 0)$ & $3.000(0 \mid 0)$ & $7.289(0 \mid 1000)$ & $4.043(0 \mid 358)$ & $3.125(0 \mid 35)$ & $3.000(0 \mid 0)$ \\
0.30 & 3 & $2.758(121 \mid 0)$ & $2.990(5 \mid 0)$ & $7.327(0 \mid 1000)$ & $4.017(0 \mid 369)$ & $3.181(0 \mid 54)$ & $2.998(1 \mid 0)$ \\
0.25 & 3 & $2.996(2 \mid 0)$ & $3.000(0 \mid 0)$ & $7.315(0 \mid 1000)$ & $3.989(0 \mid 363)$ & $3.162(0 \mid 55)$ & $3.000(0 \mid 0)$ \\
0.20 & 3 & $2.754(123 \mid 0)$ & $2.998(1 \mid 0)$ & $7.321(0 \mid 1000)$ & $4.140(0 \mid 397)$ & $3.189(0 \mid 56)$ & $3.000(0 \mid 0)$ \\
0.15 & 3 & $2.324(338 \mid 0)$ & $2.978(11 \mid 0)$ & $7.360(0 \mid 1000)$ & $4.100(0 \mid 389)$ & $3.190(0 \mid 54)$ & $3.000(0 \mid 0)$ \\
0.10 & 3 & $1.508(746 \mid 0)$ & $2.878(61 \mid 0)$ & $7.269(0 \mid 1000)$ & $4.210(0 \mid 437)$ & $3.163(0 \mid 51)$ & $2.994(3 \mid 0)$ \\
\hline
\end{tabular}

${ }^{\dagger}$ Where $N=T=100, r=3, \theta=1, k \max =8, \rho=0.5, \beta=0.2, J=\max \{10, N / 20\}$,

$F_{1}, F_{2} \sim N(0,1)$, and $F_{3} \sim N\left(0, S N R_{3}\right)$.

Table 2 reports the results from the first case of the second part of our simulations. In short, the $\hat{r}_{T C R}, \hat{r}_{E R}$ and $\hat{r}_{G R}$ estimators are better than the other estimators. However, when $N=T \leq 100$, all the estimators are bad; 
when $N=T \geq 125$, the $\hat{r}_{T C R}, \hat{r}_{E R}, \hat{r}_{G R}$ estimators begin to perform well.

Moreover, with increasing $N(=T), \hat{r}_{T C R}$ estimator outperforms $\hat{r}_{E R}$ and $\hat{r}_{G R}$ estimators. Table 3 reports the results from the second case of the second part of our simulations. With one factor turning weaker, the $\hat{r}_{T C R}$ and $\hat{r}_{G R}$ estimators perform better than the other estimators. In fact, the $\hat{r}_{T C R}$ estimator is also slightly better than the $\hat{r}_{G R}$ estimator.

Table 4: Estimation with different values of $k \max$ with three factors

\begin{tabular}{cccccccc}
\hline$k \max$ & $r$ & $\hat{r}_{E R}$ & $\hat{r}_{G R}$ & $\hat{r}_{B N}$ & $\hat{r}_{A B C}$ & $\hat{r}_{E D}$ & $\hat{r}_{T C R}$ \\
\hline 8 & 3 & $3.000(0 \mid 0)$ & $3.000(0 \mid 0)$ & $7.215(0 \mid 1000)$ & $3.198(0 \mid 106)$ & $3.006(0 \mid 6)$ & $3.000(0 \mid 0)$ \\
12 & 3 & $3.000(0 \mid 0)$ & $3.000(0 \mid 0)$ & $9.747(0 \mid 1000)$ & $5.376(0 \mid 386)$ & $3.157(0 \mid 37)$ & $3.000(0 \mid 0)$ \\
16 & 3 & $3.000(0 \mid 0)$ & $3.000(0 \mid 0)$ & $10.92(0 \mid 1000)$ & $10.08(0 \mid 790)$ & $4.028(0 \mid 109)$ & $3.000(0 \mid 0)$ \\
20 & 3 & $3.000(0 \mid 0)$ & $3.000(0 \mid 0)$ & $11.82(0 \mid 1000)$ & $10.25(0 \mid 713)$ & $4.173(0 \mid 125)$ & $3.000(0 \mid 0)$ \\
25 & 3 & $3.000(0 \mid 0)$ & $3.000(0 \mid 0)$ & $12.77(0 \mid 1000)$ & $10.28(0 \mid 800)$ & $4.155(0 \mid 120)$ & $3.000(0 \mid 0)$ \\
30 & 3 & $3.000(0 \mid 0)$ & $3.000(0 \mid 0)$ & $13.58(0 \mid 1000)$ & $10.16(0 \mid 781)$ & $4.298(0 \mid 135)$ & $3.000(0 \mid 0)$ \\
\hline
\end{tabular}

${ }^{\dagger}$ Where $N=T=150, r=3, \theta=1, \rho=0.5, \beta=0.2, J=\max \{10, N / 20\}$, and $F_{1}, F_{2}, F_{3} \sim N(0,1)$.

In the third part, we investigate how the choice of large kmax may influence the performances of estimators when the eigenvalues $\tilde{\mu}_{N T, i}$ are close to zero for some large $i(<m)$. Six different values are used for $k$ max, and the data generating process is the same as the case $C_{4}$ of the first part with $N=T=$ 150, the estimated results are recorded in Table 4 . The results show that the performances of the TCR estimator are the same as those of the ER and GR estimators, which are insensitive to kmax. The ED estimator is less sensitive to kmax than the $\mathrm{ABC}$ and $\mathrm{BIC} 3$ estimators, but the BIC3 estimator is easily sensitive to $k \max$, because the mean of the estimations in the BIC3 estimator increases with kmax obviously.

The fourth part of our simulations considers the case in which both factors have strong explanatory power, but one factors power is increasingly dominant. We generate data using two factors with different $S N R \mathrm{~s}\left(S N R_{1}=1, S N R_{2}>\right.$ 1), i.e., the two factors are drawn from $N(0,1)$ and $N\left(0, S N R_{2}\right)$, respectively. We investigate four cases $C_{5}: N=T=75 ; C_{6}: N=T=100 ; C_{7}: N=T=$ 150; $C_{8}: N=T=200$. Also, we can examine how large a difference in the 

the true number of factors.

Table 5 reports the results of the six estimators when there is one dominant factor. For such a case with large $S N R_{2}$, the TCR and GR estimators are always outperforming the other estimators, the peak of ER's estimations may be often at 1. Further, the TCR estimator perform better than the GR estimator in most cases with large $S N R_{2}$ and $N=T \leq 150$.

Table 5: Effects of dominant factor with two factors

\begin{tabular}{|c|c|c|c|c|c|c|c|c|}
\hline & $S N R_{2}$ & $r$ & $\hat{r}_{E R}$ & $\hat{r}_{G R}$ & $\hat{r}_{B N}$ & $\hat{r}_{A B C}$ & $\hat{r}_{E D}$ & $\hat{r}_{T C R}$ \\
\hline \multirow{6}{*}{$C_{5}$} & 1 & 2 & $2.009(8 \mid 6)$ & $2.136(2 \mid 36)$ & $6.162(0 \mid 1000)$ & $6.391(0 \mid 973)$ & $5.045(0 \mid 644)$ & $2.397(1 \mid 95)$ \\
\hline & 3 & 2 & $1.874(130 \mid 1)$ & $2.005(21 \mid 7)$ & $6.170(0 \mid 1000)$ & $6.264(0 \mid 960)$ & $5.133(0 \mid 664)$ & $2.231(5 \mid 58)$ \\
\hline & 7 & 2 & $1.652(348 \mid 0)$ & $1.985(15 \mid 0)$ & $6.184(0 \mid 1000)$ & $6.396(0 \mid 969)$ & $5.025(0 \mid 634)$ & $2.022(0 \mid 5)$ \\
\hline & 10 & 2 & $1.449(551 \mid 0)$ & $1.975(25 \mid 0)$ & $6.155(0 \mid 1000)$ & $6.325(0 \mid 981)$ & $4.975(0 \mid 631)$ & $2.024(0 \mid 5)$ \\
\hline & 15 & 2 & $1.004(996 \mid 0)$ & $1.506(494 \mid 0)$ & $6.170(0 \mid 1000)$ & $6.3270 \mid 960)$ & $5.015(0 \mid 630)$ & $2.084(57 \mid 35)$ \\
\hline & 20 & 2 & $1.000(1000 \mid 0)$ & $1.254(746 \mid 0)$ & $6.136(0 \mid 1000)$ & $6.290(0 \mid 966)$ & $5.034(0 \mid 641)$ & $1.981(89 \mid 18)$ \\
\hline \multirow{6}{*}{$C_{6}$} & 1 & 2 & $2.000(0 \mid 0)$ & $2.000(0 \mid 0)$ & $6.768(0 \mid 1000)$ & $4.320(0 \mid 613)$ & $2.858(0 \mid 175)$ & $2.000(0 \mid 0)$ \\
\hline & 3 & 2 & $1.944(56 \mid 0)$ & $1.965(35 \mid 0)$ & $6.761(0 \mid 1000)$ & $4.460(0 \mid 620)$ & $2.751(0 \mid 149)$ & $2.000(0 \mid 0)$ \\
\hline & 7 & 2 & $1.368(632 \mid 0)$ & $1.999(1 \mid 0)$ & $6.759(0 \mid 1000)$ & $4.390(0 \mid 599)$ & $2.751(0 \mid 148)$ & $1.999(1 \mid 0)$ \\
\hline & 10 & 2 & $1.092(908 \mid 0)$ & $1.934(66 \mid 0)$ & $6.819(0 \mid 1000)$ & $4.234(0 \mid 555)$ & $2.755(0 \mid 145)$ & $1.998(2 \mid 0)$ \\
\hline & 15 & 2 & $1.004(996 \mid 0)$ & $1.850(150 \mid 0)$ & $6.813(0 \mid 1000)$ & $4.281(0 \mid 586)$ & $2.737(0 \mid 149)$ & $1.999(1 \mid 0)$ \\
\hline & 20 & 2 & $1.000(1000 \mid 0)$ & $1.741(259 \mid 0)$ & $6.800(0 \mid 1000)$ & $4.232(0 \mid 569)$ & $2.773(0 \mid 154)$ & $1.998(2 \mid 0)$ \\
\hline \multirow{6}{*}{$C_{7}$} & 1 & 2 & $2.000(0 \mid 0)$ & $2.000(0 \mid 0)$ & $7.058(0 \mid 1000)$ & $2.334(0 \mid 134)$ & $2.019(0 \mid 17)$ & $2.000(0 \mid 0)$ \\
\hline & 3 & 2 & $2.000(0 \mid 0)$ & $2.000(0 \mid 0)$ & $7.058(0 \mid 1000)$ & $2.353(0 \mid 135)$ & $2.018(0 \mid 14)$ & $2.000(0 \mid 0)$ \\
\hline & 7 & 2 & $1.511(489 \mid 0)$ & $1.999(1 \mid 0)$ & 7.037(0|1000) & $2.318(0 \mid 137)$ & $2.013(0 \mid 37)$ & $2.000(0 \mid 0)$ \\
\hline & 10 & 2 & $1.047(953 \mid 0)$ & $1.994(4 \mid 0)$ & $7.046(0 \mid 1000)$ & $2.284(0 \mid 111)$ & $2.021(0 \mid 16)$ & $2.000(0 \mid 0)$ \\
\hline & 15 & 2 & $1.000(1000 \mid 0)$ & $1.797(203 \mid 0)$ & $7.029(0 \mid 1000)$ & $2.286(0 \mid 110)$ & $2.016(0 \mid 11)$ & $2.000(0 \mid 0)$ \\
\hline & 20 & 2 & $1.000(1000 \mid 0)$ & $1.963(37 \mid 0)$ & $6.997(0 \mid 1000)$ & $2.357(0 \mid 125)$ & $2.016(0 \mid 14)$ & $2.000(0 \mid 0)$ \\
\hline \multirow{6}{*}{$C_{8}$} & 1 & 2 & $2.000(0 \mid 0)$ & $2.000(0 \mid 0)$ & $6.750(0 \mid 1000)$ & $2.046(0 \mid 27)$ & $2.007(0 \mid 7)$ & $2.000(0 \mid 0)$ \\
\hline & 3 & 2 & $2.000(0 \mid 0)$ & $2.000(0 \mid 0)$ & $6.725(0 \mid 1000)$ & $2.073(0 \mid 44)$ & $2.011(0 \mid 10)$ & $2.000(0 \mid 0)$ \\
\hline & 7 & 2 & $1.411(589 \mid 0)$ & $2.000(0 \mid 0)$ & $6.704(0 \mid 1000)$ & $2.054(0 \mid 37)$ & $2.003(0 \mid 3)$ & $2.000(0 \mid 0)$ \\
\hline & 10 & 2 & $1.460(540 \mid 0)$ & $2.000(0 \mid 0)$ & $6.705(0 \mid 1000)$ & $2.049(0 \mid 34)$ & $2.010(0 \mid 4)$ & $2.000(0 \mid 0)$ \\
\hline & 15 & 2 & $1.558(442 \mid 0)$ & $2.000(0 \mid 0)$ & $6.703(0 \mid 1000)$ & $2.036(0 \mid 25)$ & $2.005(0 \mid 5)$ & $2.000(0 \mid 0)$ \\
\hline & 20 & 2 & $1.047(953 \mid 0)$ & $2.000(0 \mid 0)$ & $6.731(0 \mid 1000)$ & $2.061(0 \mid 39)$ & $2.015(0 \mid 15)$ & $2.000(0 \mid 0)$ \\
\hline \multicolumn{9}{|c|}{$\begin{array}{l}\dagger \text { Where } r=2, \theta=1, k \max =8, \rho=0.5, \beta=0.2, J=\max \{10, N / 20\}, F_{1} \sim N(0,1) \text {, } \\
\text { and } F_{2} \sim N\left(0, S N R_{2}\right) . C_{5}: N=T=75 ; C_{6}: N=T=100 ; C_{7}: N=T=150 ; C_{8} \text { : }\end{array}$} \\
\hline
\end{tabular}

\section{A Real Data Example}


The real data set we use is the FRED-MD dataset updated to the more recent

Moreover, the TCR estimator outperforms the other estimators for the cases in which some factors are strongly dominant, and the cases in which both strong and weak factors are existent.

\section{Appendix: Technical Details}

The proof of Theorem 1 Under Assumptions A-C, it follows from Lemma A.11 of Ahn and Horenstein (2013) that, in probability, for any $i \leq r, \tilde{\mu}_{N T, i} \rightarrow$ 
$\mu_{N T, i}>c_{0}$ for some positive constant $c_{0}$.

Also, it follows from Lemma A.9 of Ahn and Horenstein (2013) that, for any $i \geq r+1, \tilde{\mu}_{N T, i} \leq \tilde{\mu}_{N T, r+1}=O_{p}\left(\frac{1}{m}\right)$.

Meanwhile, by Lemma A.12 of Ahn and Horenstein (2013), it holds that $V_{r+1}=O_{p}(1)$.

According to the inequality

$$
\frac{c}{1+c}<\ln (1+c)<c, \quad \text { for } \quad c \in(0, \infty),
$$

for one hand, we have that

$$
\begin{aligned}
\frac{\ln \left(1+\tilde{\mu}_{N T, i} / V_{i-1}\right)}{\ln \left(1+\tilde{\mu}_{N T, i+1} / V_{i}\right)} & <\frac{\tilde{\mu}_{N T, i} / V_{i-1}}{\left(\tilde{\mu}_{N T, i+1} / V_{i}\right) /\left(1+\tilde{\mu}_{N T, i+1} / V_{i}\right)} \\
& =\frac{\tilde{\mu}_{N T, i}}{\tilde{\mu}_{N T, i+1}} \cdot \frac{V_{i}+\tilde{\mu}_{N T, i+1}}{V_{i}+\tilde{\mu}_{N T, i}}=O_{p}(1),
\end{aligned}
$$

for $i=1,2, \ldots, r-1, r+1, \ldots, k \max$. For another,

$$
\frac{V_{r}}{V_{r-1}+\tilde{\mu}_{N T, r}}=\frac{V_{r+1}+\tilde{\mu}_{N T, r+1}}{V_{r+1}+2 \tilde{\mu}_{N T, r}+\tilde{\mu}_{N T, r+1}}=O_{p}(1),
$$

295

then, we have

$$
\begin{aligned}
\frac{\ln \left(1+\tilde{\mu}_{N T, r} / V_{r-1}\right)}{\ln \left(1+\tilde{\mu}_{N T, r+1} / V_{r}\right)} & >\frac{\left(\tilde{\mu}_{N T, r} / V_{r-1}\right) /\left(1+\tilde{\mu}_{N T, r} / V_{r-1}\right)}{\tilde{\mu}_{N T, r+1} / V_{r}} \\
& =\frac{\tilde{\mu}_{N T, r}}{\tilde{\mu}_{N T, r+1}} \cdot \frac{V_{r}}{V_{r-1}+\tilde{\mu}_{N T, r}}=O_{p}(m) .
\end{aligned}
$$

It then follows that

$$
\lim _{m \rightarrow \infty} \operatorname{Pr}\left(\hat{r}_{T C R}=r\right)=1 .
$$

This means the consistency of the estimator $\hat{r}_{T C R}$, and then the proof of Theorem 1 is completed.

\section{Acknowledgments}

We thank the Editor, an Associate Editor and two anonymous referees for valuable comments that led to the substantial improvement of this paper. The research of Rubing Liang was supported in part by National Science Foundation of Guangdong Province of China (No.2015A030310365). The research of 
Qiang Xia was supported in part by National Science Foundation of Guangdong Province of China (No.2016A030313414) and National statistical plan for scientific research project of China (No.2015LZ48). The research of Jianhong Wu was supported in part by National Science Foundation of China (No.11671263).

\section{References}

Ahn, S.C., Horenstein, A.R., 2013. Eigenvalue ratio test for the number of factors. Econometrica 81, 1203-1227.

Alessi, L., Barigozzi, M., Capasso, M., 2010. Improved penalization for determining the number of factors in approximate factor models. Statistics and Probability Letters 80, 1806-1813.

Amengual, D., Watson, M., 2007. Consistent estimation of the number of dynamic factors in a large $\mathrm{n}$ and $\mathrm{t}$ panel. Journal of Business and Economic Statistics 25, 91-96.

Bai, J., Ng, S., 2002. Determining the number of factors in approximate factor models. Econometrica 70, 191-221.

Bai, J., Ng, S., 2007. Determining the number of primitive shocks in factor models. Journal of Business and Economic Statistics 25, 52-60.

320 Chamberlain, G., Rothschild, M., 1983. Arbitrary, factor structure, and meanvariance analysis on large asset markets. Econometrica 51, 1281-1304.

Fan, J., Liao, Y., Mincheva, M., 2013. Large covariance estimation by thresholding principal orthogonal complements. Journal of the Royal Statistical Society: Series B 75, 603-680.

325 Forni, M., Hallin, M., Lippi, M., Reichlin, L., 2000. The generalized dynamicfactor model: identification and estimation. The Review of Economics and Statistics 82, 540-554. 
Hallin, M., Liska, R., 2007. Determining the number of factors in the general dynamic factor model. Journal of the American Statistical Association 102, 603-617.

Lam, C., Yao, Q., 2012. Factor modeling for high-dimensional time series: inference for the number of factors. The Annals of Statistics 40, 694-726.

McCracken, M., Ng, S., 2015. Fred-md: a monthly database for macroeconomic research. Journal of Business and Economic Statistics 34, 574-589.

Onatski, A., 2006. Determining the number of factors from empirical distribution of eigenvalues. Working Paper, Columbia University.

Onatski, A., 2009. Testing hypotheses about the number of factors in large factor models. Econometrica 77, 1447-1479.

Onatski, A., 2010. Determining the number of factors from empirical distribution of eigenvalues. Review of Economic and Statistics 92, 1004-1016.

Pan, J., Yao, Q., 2008. Modelling multiple time series via common factors. Biometrika 95, 356-379.

Stock, J., Watson, M., 2002. Forecasting using principal components from a large number of predictors. Journal of the American statistical association 97, 1167-1179.

Xia, Q., Xu, W., Zhu, L.X., 2015. Consistently determining the number of factors in multivariate volatility modelling. Statistica Sinica 25, 1025-1044. 